\title{
Calculation of the Transmission Spectrum of Long-Period Fiber Grating Based on the Mode Field Analysis Method
}

\author{
Wei Zhang \\ School of automobile engineering, Linyi University, Linyi City Shandong \\ 276005, China \\ email: zhwei-107@163.com
}

\begin{abstract}
By using the coupled mode theory, the vector expressions of the core and the cladding electromagnetic field components of long-period fiber grating are analyzed. After the effective refractive indexes and the coupling constants of the core and cladding mode are solved, the transmission spectrum of the long-period fiber grating is obtained through solving the coupled mode equations by numerical integration method. The transmission spectra corresponding to single-mode and multi-mode are simulated, respectively. The research provides the theoretical foundation for the optimization design of the long-period fiber grating and its application in the field of the optic fiber sensing and communication.
\end{abstract}

Keywords: Coupled-mode theory; mode field analysis method; long-period fiber grating; transmission spectrum; coupling constant

\section{Introduction}

By virtue of advantages such as anti-electromagnetic interference, high precision, good stability, anti-corrosion, low insertion loss, low-level back reflection, small size, easy of mass-fabrication and so on, long-period fiber gratings (LPFGs) have attracted much attention, and become the key study subject in the field of sensing and communication in recent years ${ }^{[1 \sim 3]}$. The LPFG can couple light from core mode to cladding modes when the phase matching condition is met, so a series of loss peaks appear in its transmission spectrum. As a new kind of important optical fiber passive component, the research on the calculation of the transmission spectrum becomes a key issue because its working principle is closely related to the resonant wavelength. Yariv proposed a formula method for calculating the transmission spectrum of the LPFG, which can be used to solve core mode coupling with single cladding mode ${ }^{[4]}$. Kermisch proposed a kind of line matrix calculation method to get the transmission spectrum of the LPFG ${ }^{[5]}$. Kogelnik deduced two coupled mode equations to a 
single Riccati differential equation to get the numerical solution of the transmission spectrum of the LPFG ${ }^{[6]}$.These calculation methods are complex in theory or calculation. In this work, Mode Field Analysis Method for calculating the transmission spectrum of the LPFG is proposed. The results will provide a high accuracy and simple calculation method for calculating LPFG transmission spectra, which is important for the research and application of LPFG.

\section{Theoretical analysis on the method for solving the transmission spectrum of the LPFG}

Figure 1(a) shows the structural diagram of the LPFG. Figure 1(b) shows the refractive index profile of the LPFG.

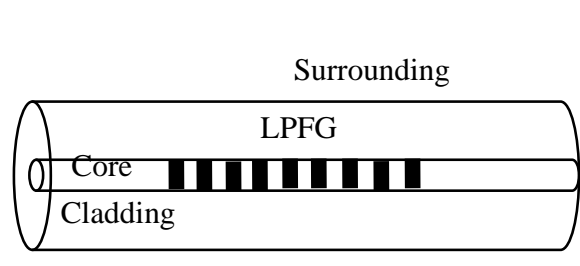

(a)

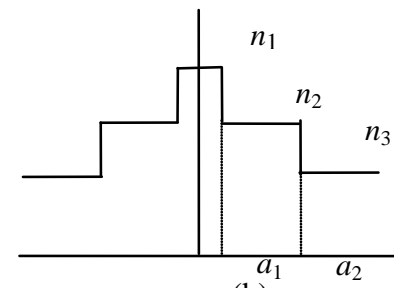

(b)

$\begin{array}{ll}\text { (a) Structure model of the LPFG } & \text { (b) Refractive index profile }\end{array}$

Fig.1 Diagram of the LPFG model

The refractive indices of the core, cladding and surrounding are $n_{1}, n_{2}, n_{3}$, respectively. $a_{1}, a_{2}$ are the radii of the core, cladding, respectively. The grating period is $\Lambda$. The average index change of grating area is $\sigma$. The order of magnitude is $10^{-4}$.

For an ideal uniform fiber, core mode and each cladding mode are orthogonal to each other, so there is no mode coupling in it. When a grating is written in the fiber, the consistency of the optical fiber properties is destroyed, and the disturbance of the dielectric constant is produced. This kind of periodic disturbance along the fiber axial makes the core mode coupled to the cladding modes. In order to make the solving of the transmission spectrum of the LPFG easy, we do the following approximation:

(1) Due to the field component along the fiber axial is smaller 1 2 orders of magnitude than that of along the fiber radial, and longitudinal coupling coefficient is smaller 2 4 orders of magnitude than that of horizontal, the longitudinal coupling coefficient can be ignored.

(2) The refractive index modulation exists only in fiber core, relativing to the coupling between the co-directional core mode and the contra-directional core mode and cladding modes, the coupling between the cladding modes can be ignored.

(3) The refractive index modulation of the LPFG axial direction is circular symmetry, so the coupling between the forward propagation of core mode $\mathrm{HE}_{11}$ and the co-propagating cladding modes $\mathrm{HE}_{1 \mathrm{v}}$ can only be considered ${ }^{[7]}$. 
By the above approximation, the calculations of the LPFG transmission spectra can be performed according to the following steps:

(1) Setting the basic parameters of the LPFG

After setting the parameters of the LPFG including the refractive indices of the core, the inner cladding, the extramural cladding, the radii of the core and cladding, the period of grating and the grating length, the variation of fiber grating core refractive index along $\mathrm{Z}$ axis $\delta_{n}(z)$ can be expressed as:

$$
\delta_{n}(z)=\bar{\delta}_{n}(z)\{1+v \cos [(2 \pi / \Lambda) z+\phi(z)]\}
$$

Where, $\bar{\delta}_{n}(z)$ is the average variation of the effective refractive index in one grating period, $v$ is the contrast of the refractive index modulation, $\Lambda$ is the period of grating, $\phi(z)$ is the phase shift of the fiber gratings related to the origin.

(2) Calculating the effective refractive index for the core mode and the cladding mode. The core mode eigenvalue equation ( $\mathrm{LP}_{01}$ mode)can be expressed as ${ }^{[7]}$ :

$$
\frac{V \sqrt{1-b} J_{1}(V \sqrt{1-b})}{J_{0} \sqrt{1-b}}=\frac{V \sqrt{b} K_{1}(V \sqrt{b})}{K_{0} \sqrt{b}}
$$

Where, $b=\left(n_{\text {eff ,co }}^{2}-n_{1}^{2}\right) /\left(n_{2}^{2}-n_{1}^{2}\right)$ is the normalized propagating constant; $V=(2 \pi / \lambda) a_{1} \sqrt{n_{1}^{2}-n_{2}^{2}}$ is the normalized frequency; $\mathrm{J}_{0}$ and $\mathrm{J}_{1}$ are 0 order and 1 order Bessel functions; $K_{0}$ and $K_{1}$ are 0 order and 1 order correction of the second class of Bessel functions. The propagating constant $\beta_{c o}$ can be obtained by putting set parameters into the core mode eigenvalue equation, and then the effective refractive index for the core mode $n_{\text {eff }, \text { co }}\left(\lambda_{i}\right)=\beta_{\text {co }}\left(\lambda_{i}\right) / k$ can be solved.

The cladding mode eigenvalue equation(dispersion equation) can be expressed as ${ }^{[8]}$ :

$$
\begin{aligned}
& z_{0}=z_{1} \\
& z_{0}=\frac{1}{s_{2}} \cdot \frac{u_{2}\left(J K+s_{1} s_{2} u_{21} u_{32} / n_{2}^{2} a_{1} a_{2}\right) p_{v}\left(a_{2}\right)-K q_{v}\left(a_{2}\right)+J r_{v}\left(a_{2}\right)-s_{v}\left(a_{2}\right) / u_{2}}{-u_{2}\left(\left(u_{32} / n_{2}^{2} a_{2}\right) J-\left(u_{21} / n_{1}^{2} a_{1}\right) K\right) p_{v}\left(a_{2}\right)+\left(u_{32} / n_{1}^{2} a_{2}\right) q_{v}\left(a_{2}\right)+\left(u_{21} / n_{1}^{2} a_{1}\right) r_{v}\left(a_{2}\right)} \\
& z_{1}=s_{1} \cdot \frac{u_{2}\left(\left(u_{32} / a_{2}\right) J-\left(n_{3}^{2} u_{21} / n_{2}^{2} a_{1}\right) K\right) p_{v}\left(a_{2}\right)-\left(u_{32} / a_{2}\right) q_{v}\left(a_{2}\right)-\left(u_{21} / a_{1}\right) r_{v}\left(a_{2}\right)}{u_{2}\left(\left(n_{3}^{2} / n_{2}^{2}\right) J K+s_{1} s_{2} u_{21} u_{32} / n_{1}^{2} a_{1} a_{2}\right) p_{v}\left(a_{2}\right)-\left(n_{3}^{2} / n_{1}^{2}\right) K q_{v}\left(a_{2}\right)+J r_{v}\left(a_{2}\right)-\left(n_{2}^{2} / n_{1}^{2} u_{2}\right) s_{v}\left(a_{1}\right)}
\end{aligned}
$$

The effective refractive index for the first-order cladding modes $n_{\text {eff,cl }}^{m}$ could be obtained by solving the above cladding mode eigenvalue equation. (For general single-mode optical fiber whose diameter is $125 \mu \mathrm{m}$ usually, there are 
hundreds of cladding modes), then the propagating constant $\beta_{c l}^{m}$ can be obtained.

(3) Solving the mode field (electric field and magnetic field) distribution of the fundamental core mode and the first-order cladding modes.

The normalized coefficient of the mode field $E_{01}^{c o}$ can be calculated according to the following expression:

$$
E_{01}^{c o} \cong\left(\frac{Z_{0} b}{\pi n_{2} \sqrt{1+2 b \Delta}}\right)^{1 / 2} \frac{1}{a_{1} J_{1}(V \sqrt{1-b})}
$$

Where, $\Delta=\left(n_{1}-n_{2}\right) / n_{1}$ is the normalized refractive index difference; $Z_{0}=\sqrt{\mu_{0} / \varepsilon_{0}}=377 \Omega ; V=u_{1}^{2}+v_{1}^{2}$ is defined as the normalized frequency, $u_{1}=a_{1} \sqrt{k^{2} n_{1}^{2}-\beta_{c o}^{2}}$ is the radial normalized phase-shifting constant, $v_{1}=a_{1} \sqrt{\beta_{c o}^{2}-k^{2} n_{2}^{2}}$ is the radial normalized attenuation constant; $k=2 \pi / \lambda$ is the wave number. Thus, $V=(2 \pi / \lambda) a_{1} \sqrt{n_{1}^{2}-n_{2}^{2}}$ can be obtained. Then the radial electric vector component $E_{r}^{c o}$ and azimuthal electric vector component $E_{\phi}^{c o}$ of the fundamental core mode can be solved; and then the radial electromagnetic field components ( $E_{r}^{c l}$ and $H_{r}^{c l}$ ) and azimuthal electromagnetic field components ( $E_{\phi}^{c l}$ and $H_{\phi}^{c l}$ ) of the core mode and the cladding modes can be solved by Debye potential energy method ${ }^{[8]}$.

(4) Calculation of the self-coupling coefficient of core-mode and the cross-coupling coefficient between core mode and cladding mode according to the modefield distributions.

The self-coupling coefficient of the fundamental core mode $k_{01-01}^{c o-c o}$ is:

$$
k_{01-01}^{c o-c o}=\sigma(z) \frac{2 \pi n_{1}^{2} b}{\lambda n_{2} \sqrt{1+2 b \Delta}}\left[1+\frac{J_{0}^{2}(V \sqrt{1-b})}{J_{1}^{2}(V \sqrt{1-b})}\right]
$$

The cross-coupling coefficient $k_{1 v-01}^{c l-c o}$ between the core mode and the cladding modes is: 


$$
\begin{aligned}
& k_{1 v-01}^{c l-c o}=\sigma(z) \frac{2 \pi}{\lambda}\left(\frac{\pi b}{Z_{0} n_{2} \sqrt{1+2 b \Delta}}\right)^{1 / 2} \frac{n_{1}^{2} u_{1}}{u_{1}^{2}-V^{2}(1-b) / a_{1}^{2}}\left(1+\frac{\sigma_{2} \zeta_{0}}{n_{1}^{2}}\right) E_{1 v}^{c l} \\
& \times\left[\frac{u_{1} J_{1}\left(u_{1} a_{1}\right) J_{0}(V \sqrt{1-b})}{J_{1}(V \sqrt{1-b})}-\frac{V \sqrt{1-b} J_{0}\left(u_{1} a_{1}\right)}{a_{1}}\right]
\end{aligned}
$$

(5) Solving the coupled-mode differential equations as ${ }^{[9]}$ :

The coupled-mode equations of long-period fiber gratings can be expressed

$$
\begin{aligned}
& \frac{d A^{c o}}{d z}=i k_{01-01}^{c o-c o} A^{c o}+i \sum_{v} \frac{m}{2} k_{1 v-01}^{c l-c o} A_{v}^{c l} \exp \left(-i 2 \delta_{1 v-01}^{c l-c o} z\right) \\
& \frac{d A_{v}^{c l}}{d z}=+i \frac{m}{2} k_{1 v-01}^{c l-c o} A^{c o} \exp \left(+i 2 \delta_{1 v-01}^{c l-c o} z\right)
\end{aligned}
$$

Where $\delta_{1 v-01}^{c l-c o}$ is a detuning parameter between the fundamental core mode and the $v$ th cladding mode:

$$
\delta_{1 v-01}^{c l-c o}=\left(\beta_{01}^{c o}-\beta_{1 v}^{c l}-2 \pi / \Lambda\right) / 2
$$

$A^{c o}$ is the amplitude of the core mode along $\mathrm{z}$ axis direction, $A_{v}^{c l}$ is the amplitude of the $v$ th cladding mode along $z$ axis direction. $\boldsymbol{k}_{01-01}^{c o-c o}$ and $\boldsymbol{k}_{1 v-01}^{c l-c o}$ are the self-coupling coefficient of core-mode and the cross-coupling coefficient between core mode and the vth cladding mode, respectively. According to (5)、(6) and (9), $A^{c o}$ and $A_{v}^{c l}$ can be solved.

(6) The transmission spectrum of the LPFG is obtained according to initial conditions and boundary conditions

Suppose the center of the grating is the origin of the z-axis, then the boundary conditions for a long-period grating are $A^{c o}(z=-L / 2)=1$ and $A_{v}^{c l}(z=-L / 2)=0$.For each identified wavelength, the transmissivity $T_{\lambda}$ of the LPFG can be expressed as:

$$
T_{\lambda}=\frac{A^{c o}(L / 2)}{A^{c o}(-L / 2)}
$$

The amplitude of the core mode along $\mathrm{z}$ axis direction $A^{c o}$ and the amplitude of the vth cladding mode along $\mathrm{z}$ axis direction $A_{v}^{\text {cl }}$ are solved by the 
numerical integral method, so this method is also named integral method. In theory this method can directly solve the total transmission spectrum of the fundamental core mode coupling with all order cladding modes, and the transmission spectrum can be obtained accurately by this method.

\section{Numerical simulation of the transmission spectrum for the LPFG}

The grating parameters used in calculation are: $a_{1}=2.625 \mu m, a_{2}=62.5 \mu m$; $\mathrm{n}_{0}=1.468, \mathrm{n}_{1}=1.458, \mathrm{n}_{2}=1.45, \mathrm{n}_{3}=1$. The average index change of grating area $\sigma=4 \times 10^{-4}$, the grating period $\Lambda=450 \mu \mathrm{m}$, the grating length $\mathrm{L}=2.0 \mathrm{~cm}$.

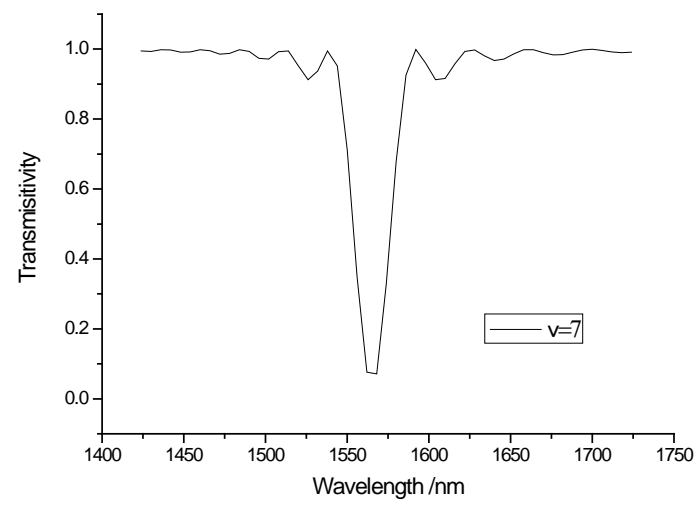

(a) Single mode $(\mathrm{v}=7)$

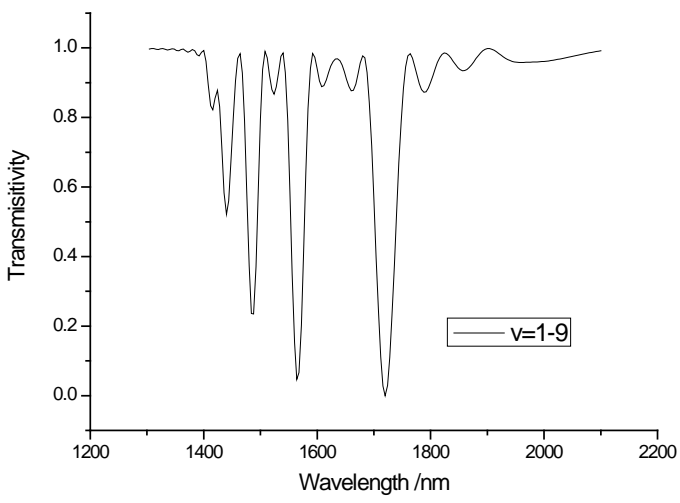

(b) Multi-mode $(\mathrm{v}=1-9)$ 
Fig.2 Transmission spectra of the LPFG solved by integral method

Figure 2(a) shows the transmission spectrum for the core mode coupling with the 7th cladding mode. Besides the maximum resonance peak appears at $\lambda=1562 \mathrm{~nm}$, other small resonance peaks appear on both sides of the maximum resonance peak. These sidelobes are inducted by the mutations of the fiber core refractive index on both ends of the fiber. The energies of these sidelobes are far less than that of the resonance peak at $\lambda=1562 \mathrm{~nm}$, so they can be neglected generally. Figure 2(b) shows the transmission spectrum of the LPFG for the core mode coupling with the multi-cladding mode orders ( $\mathrm{v}$ 错误!未定义书签。=1-9). We can see that a series of discrete loss peaks appear in the transmission spectrum. Each loss peak corresponds to one odd number cladding mode coupling with the core mode, those loss peaks corresponding to the core mode coupling with the even number cladding modes are so small that almost invisible to our eyes. The intervals between adjacent loss peaks and the transmissivities increase with the increase of the cladding mode. The reason for this is that the energy coupled to the cladding modes is recoupled back to the corn mode partly ${ }^{[10]}$, so the energy of the cladding modes decreases rapidly with the increase of the cladding mode order. In addition, for the higher order cladding modes, the coupling strength is very weak ${ }^{[11]}$, which causes the loss peaks so small that could not be observed in the transmission spectrum.

\section{Summary}

Based on the vector field analysis method, a numerical model for three-cladding long-period fiber gratings is established by using the coupled-mode theory. The transmission spectra of the long-period fiber gratings are analyzed and calculated. At first, the electric and magnetic fields within the fiber core and cladding are analyzed by means of vector analysis, the component of the vector field of the fiber core, cladding, thin films, and external environment are calculated, respectivily. Then the coupling constant and the effective refractive index of the fiber core and cladding mode are acquired. Finally, the amplitude of fiber core and cladding mode are worked out by the integration of the coupled-mode equations, then the transmissivity of the long-period fiber grating is obtained. The research can provide some theory basis for the optimization design of the long-period fiber grating and its actual application in the field of optical fiber communication.

\section{Acknowledgments}

This work was jointly supported by Science and Technology Development Plan Project of Shandong Province (Grant No.2012YD01051) and Technology Development Plan Project of Linyi (Grant No.613021). 


\section{References}

[1] Stephen W J, Ralph P T: Optical fibre long-period grating sensors: characteristics and application, Meas. Sci. Technol, 2003, 15(5): R49-R61.

[2] Han M, Wang Y J, Wang Y M, et al: Fiber-optic physical and biochemical sensing based on transient and traveling long-period gratings. Opt. Lett, 2009, 34 (1): 100-102.

[3] Gwandu B A, Shu X, Allsop T D P, et al: Simultaneous refractive index and temperature measurement using cascaded long period fiber grating in double-cladding fibre. Electronics Lett., 2002, 38(14.4): 686-695.

[4] A.Yariv: Coupled-mode theory for guided-wave optics, IEEE J. Quantum Electron.QE-9 (1973) 919-933.

[5] H. Kogelnik: Filter response of nonuniform almost-periodic structures, Bell Syst.Tech. J. 1976,1 (55) :109-126.

[6] D. Kermisch: Nonuniform sinusoidally modulated dielectric grating, J. Opt. Soc.Am. 1969, 11 (59) :1409-1414.

[7] T. Erdogan: Fiber grating spectra. J. Light. Technol. 1997, 15 (8):1277-1294.

[8] C. Tsao, in: Optical Fiber Waveguide Analysis, Oxford University Press, New York,1992.

[9] T. Erdogan: Cladding-mode resonances in short-and long period fiber grating filters. Opt. Soc. Am. A/ Vol. 14, 1997,(8):1760-1783.

[10] Y. Yang, Z. Gu: Temperature and bending simultaneous sensing properties of cascaded long- and short-period gratings, Applied Optics, 2014, 53(2):165-173.

[11] F. Abrishamian, Y. Nakai, S. Sato, and M. Imai: An efficient approach for calculating the reflection and transmission spectra of fiber Bragg gratings with acoustically induced microbending, Opt. Fiber Technol. 2007,13: 32-38. 